\title{
Correction to: Occupational and Environmental Safety and Health III
}

\author{
Pedro M. Arezes, J. Santos Baptista, Paula Carneiro, \\ Jacqueline Castelo Branco, Nélson Costa, J. Duarte, J. C. Guedes, \\ Rui B. Melo, A. Sérgio Miguel, and Gonçalo Perestrelo
}

\section{Correction to: \\ P. M. Arezes et al. (eds.), Occupational and Environmental Safety and Health III, Studies in Systems, Decision and Control 406, https://doi.org/10.1007/978-3-030-89617-1}

In the original version of the book, the following corrections have been incorporated:

In Chapter 5 "Analysis of Safety Culture Maturity in Two Finnish Companies", the first author name was corrected from Julius Pirhonena to Julius Pirhonen. The corresponding chapter author has been changed to 'Sari Tappura' and the email address was corrected from saritappura@tuni.fi to sari.tappura@tuni.fi

In Chapter 16 "Long-Term Driving Causes Gait Plantar Pressure Alterations in Subjects Groups", section “3.2 Plantar Pressure Distribution” was included.

In Chapter 22 "Cortisol as a Biomarker of Work-Related Stress in Firefighters: A Systematic Review", the author list was updated with the correct information (name, affiliations and email address) of the second author, Joana Santos.

In Chapter 42 "Ergonomics and Safety in the Design of Industrial Collaborative Robotics", the affiliations of all authors were revised and updated with the correct information. Moreover, the corresponding author was changed to Joana Santos.

The chapters and the book have been updated with the above-mentioned changes.

The updated versions of these chapters can be found at https://doi.org/10.1007/978-3-030-89617-1_5

https://doi.org/10.1007/978-3-030-89617-1_16

https://doi.org/10.1007/978-3-030-89617-1_22

https://doi.org/10.1007/978-3-030-89617-1_42

(C) The Author(s), under exclusive license to Springer Nature Switzerland AG 2022 